\title{
VECTOR-VALUED MEANS AND WEAKLY ALMOST PERIODIC FUNCTIONS
}

\author{
CHUANYI ZHANG
}

Department of Mathematics

University of British Columbia

Vancouver, B.C., Canada V6T 1 Z2

(Received June 30, 1992 and in revised form November 7, 1992)

Abstract. A formula is set up between a vector-valued mean and scalar-valued means that enbles us translate many important results about scalar-valued means developed in [1] to vector-valued means. As applications of the theory of vector-valued means, we show that the definitions of a mean in [2] and [3] are equivalent and the space of vector-valued weakly almost periodic functions is admissible.

KEY WORDS AND PHRASES. Means, semigroup, weakly almost periodic functions 1991 AMS SUBJECT CLASSIFICATION CODES. Primary 43A07; secondary 43A60

Scalar-valued means have been much studied. However, little has been done on the vector-valued means. In this paper we develop the theory of vector-valued means.

In Lemma 1.4, we set up a formula between a vector-valued mean and scalar-valued means, by which we will be able to translate many important results about scalar-valued means developed in [1] to vector-valued means. We present these results in Sections 1, 2 and 3. As an application of the theory established in these sections, we investigate vector-valued weakly almost periodic functions in Section 4.

\section{$\S 1$. Means on a Linear Subspace of $\mathcal{B}(S, X)$}

Throughout this paper, $S$ denotes a semigroup which need not have an identity, $X$ denotes a Banach space and $X^{*}$ is the dual space of $X . \mathcal{B}(S, X)$ denotes all of the bounded functions from $S$ to $X$. When $X=\mathbb{C}$, we simply write $\mathcal{B}(S)$ for $\mathcal{B}(S, X)$. $\mathcal{A}$ denotes a linear subspace of $\mathcal{B}(S, X)$ containing the constant functions. $\mathcal{L}(\mathcal{A}, X)$ denotes all of the bounded linear mappings from $\mathcal{A}$ to $X$.

Let $f \in \mathcal{B}(S, X)$. Then the right (respectively, left) translate $R_{s} f$ of $f$ by $s \in S$ is the $\operatorname{map} R_{s} f(t)=f(t s)\left(\right.$ respectively, $\left.L_{s} f(t)=f(s t)\right)$ for all $t \in S$.

$\mathcal{A}$ is said to be right (respectively, left) translation invariant if $R_{S} \mathcal{A}=\left\{R_{s} f: s \in\right.$ $S, f \in \mathcal{A}\} \subset \mathcal{A}$ (respectively, $\left.L_{S} \mathcal{A}=\left\{L_{s} f: s \in S, f \in \mathcal{A}\right\} \subset \mathcal{A}\right) . \mathcal{A}$ is said to be translation invariant if it is both right and left translation invariant.

Definition 1.1 [2]. A linear mapping $\mu: \mathcal{A} \rightarrow X$ is called a mean on $\mathcal{A}$ provided $\mu(f) \in \overline{c o} f(S)$, for all $f \in \mathcal{A}$. Denote by $M(\mathcal{A})$ the set of all means on $\mathcal{A}$.

If $\mathcal{A}$ is right (respectively, left) translation invariant, $\mu$ is said to be right (respectively, left) invariant if $\mu\left(R_{s} f\right)=\mu(f)$ (respectively, $\mu\left(L_{s} f\right)=\mu(f)$ ) for all $s \in S$ and $f \in \mathcal{A}$. 
Remark 1.2. It follows from [1. 2.1.2] that Definition 1.1 will reduce to the definition of a scalar valued mean when $X=\mathbb{C}$.

Of course, the evaluation mapping $\epsilon: S \rightarrow \mathcal{L}(\mathcal{A}, X)$, defined by

$$
\epsilon(s)(f)=f(s) \quad(s \in S, f \in \mathcal{A})
$$

is in $M(\mathcal{A})$, and if $\mu \in M(\mathcal{A})$ and $f \in \mathcal{A}$ is a constant function, then $\mu(f)$ is the constant.

The following proposition is obvious.

Proposition 1.3. If $\mathcal{A}$ is a linear subspace of $\mathcal{B}(S, X)$ containing the constant functions, then each $\mu \in M(\mathcal{A})$ is in $\mathcal{L}(\mathcal{A}, X)$ with $\|\mu\|=1$.

For each $x^{*} \in X^{*}$,

$$
x^{*} \mathcal{A}=\left\{x^{*} f=x^{*} \circ f: f \in \mathcal{A}\right\}
$$

is a linear subspace of $\mathcal{B}(S)$.

Here we have adopted the definition in [2] of a mean on $\mathcal{A}$. [3] gives a definition of a mean in terms of a scalar-valued mean on $\overline{s p}\left(X^{*} \circ \mathcal{A}\right)=\overline{s p}\left\{x^{*} \mathcal{A}: x^{*} \in X^{*}\right\}$. In the next lemma, we set up a connection like this, and we will show in Theorem 1.7 that the definitions of a mean in [2] and [3] are equivalent. We will deal with other applications in $\S 4$.

Lemma 1.4. Let $\mathcal{A}$ be a linear subspace of $\mathcal{B}(S, X)$. A mapping $\mu: \mathcal{A} \rightarrow X$ is in $M(\mathcal{A})$ if and only if, for each $x^{*} \in X^{*}$, there is a $\varphi_{\mu, x^{*}} \in M\left(x^{*} \mathcal{A}\right)$ such that

$$
x^{*} \mu(f)=\varphi_{\mu, x^{*}}\left(x^{*} f\right) \quad(f \in \mathcal{A}) .
$$

If $\mathcal{A}$ is right (left) translation invariant, then $\mu$ is right (left) invariant if and only if the $\varphi_{\mu, x^{*}}$ 's are right (left) invariant. Furthermore, the set $\varphi_{\mu}=\left\{\varphi_{\mu, x^{*}}: x^{*} \in X^{*}\right\}$ is uniquely determined by $\mu$, i.e., $\varphi_{\mu, x^{*}}=\varphi_{\mu^{\prime}, x^{*}}$ for all $x^{*} \in X^{*}$ if and only if $\mu=\mu^{\prime}$.

Proof. Sufficiency. First, $\mu$ is a linear mapping from $\mathcal{A}$ to $X$. In fact, for $f, g \in \mathcal{A}$ and $\alpha, \beta \in \mathbb{C}$,

$$
\begin{aligned}
x^{*} \mu(\alpha f+\beta g) & =\varphi_{\mu, x^{*}}\left(x^{*}(\alpha f+\beta g)\right) \\
& =\varphi_{\mu, x^{*}}\left(x^{*}(\alpha f)\right)+\varphi_{\mu, x^{*}}\left(x^{*}(\beta g)\right) \\
& =\alpha \varphi_{\mu, x^{*}}\left(x^{*} f\right)+\beta \varphi_{\mu, x^{*}}\left(x^{*} g\right) \\
& =\alpha x^{*} \mu(f)+\beta x^{*} \mu(g) \\
& =x^{*}(\alpha \mu(f)+\beta \mu(g)) .
\end{aligned}
$$

The equality is true for all $x^{*} \in X^{*}$, therefore

$$
\mu(\alpha f+\beta g)=\alpha \mu(f)+\beta \mu(g) .
$$

We claim that $\mu(f) \in \overline{c o} f(S)$, for all $f \in \mathcal{A}$. If it is not true for some $f \in \mathcal{A}$, by the Hahn-Banach theorem there is an $x^{*} \in X^{*}$ such that 


$$
\left|x^{*} \mu(f)\right|>\sup _{s \in S}\left|x^{*} f(s)\right|=\left\|x^{*} f\right\| .
$$

It follows from Remark 1.2 and Proposition 1.3 that $\varphi_{\mu, x^{*}} \in M\left(x^{*} \mathcal{A}\right)$ is in $\left(x^{*} \mathcal{A}\right)^{*}$ with $\left\|\varphi_{\mu, x}\right\|=1$. So

$$
\left|x^{*} \mu(f)\right|=\left|\varphi_{\mu, x^{*}}\left(x^{*} f\right)\right| \leq\left\|x^{*} f\right\|
$$

a contradiction.

Necessity. For each $x^{*} \in X^{*}$, define $\varphi_{\mu, x^{*}} \in\left(x^{*} \mathcal{A}\right)^{*}$ by

$$
\varphi_{\mu, x^{*}}\left(x^{*} f\right)=x^{*} \mu(f) \quad(f \in \mathcal{A}) .
$$

$\varphi_{\mu, x^{*}}$ is well-defined on $x^{*} \mathcal{A}$. For, if $x^{*} f=0$ for some $f \in \mathcal{A}$, then $f(S) \subset N\left(x^{*}\right)$, the null subspace of $x^{*}$, so $\varphi_{\mu, x^{*}}\left(x^{*} f\right)=x^{*} \mu(f)=0$ since $\mu(f) \in \overline{c o} f(S)$ (Definition 1.1). Clearly $\varphi_{\mu, x^{*}}$ is linear on $x^{*} \mathcal{A}$. Furthermore

$$
\varphi_{\mu, x^{*}}\left(x^{*} f\right)=x^{*} \mu(f) \in x^{*} \overline{c o}(f)(S) \subset \overline{c o} x^{*} f(S)
$$

so $\varphi_{\mu, x^{*}}$ is in $M\left(x^{*} \mathcal{A}\right)$.

The rest of the lemma is clear.

We can furnish $\mathcal{L}(\mathcal{A}, X)$ with two topologies, both of which make $\mathcal{L}(\mathcal{A}, X)$ a locally convex topological space. One is the strong operator topology $\tau_{s}$, which is the weakest topology of $\mathcal{L}(\mathcal{A}, X)$ relative to which the mapping $U \rightarrow U f: \mathcal{L}(\mathcal{A}, X) \rightarrow X$ is continuous for each $f \in \mathcal{A}$, and the other is the weak operator topology $\tau_{w}$, which is the weakest topology of $\mathcal{L}(\mathcal{A}, X)$ relative to which the mapping $U \rightarrow x^{*} U f: \mathcal{L}(A, X) \rightarrow \mathbb{C}$ is continuous for each $f \in \mathcal{A}$ and $x^{*} \in X^{*}$. These topologies can be relativized to $M(\mathcal{A}) \subset \mathcal{L}(\mathcal{A}, X)$.

Proposition 1.5. Let $\mathcal{A}$ be a linear subspace of $\mathcal{B}(S, X)$. Then, for $\tau_{s}$

(1) $M(\mathcal{A})$ is convex and closed in $\mathcal{L}(\mathcal{A}, X)$;

(2) $\operatorname{co}(\epsilon(S))$ is dense in $M(\mathcal{A})$;

(3) if $S$ is a topological space and $\mathcal{A} \subset \mathcal{C}(S, X)$, then $\epsilon: S \rightarrow M(\mathcal{A})$ is continuous.

Furthermore, if the range $f(S)$ of $f$ is relatively compact in $X$ for each $f \in \mathcal{A}$, then $M(\mathcal{A})$ is $\tau_{s}$-compact.

Proof.

(1) The convexity of $M(\mathcal{A})$ follows directly from Definition 1.1. To show that $M(\mathcal{A})$ is closed, let $\left\{\mu_{\alpha}\right\} \subset M(\mathcal{A})$ converge to $\mu \in \mathcal{L}(\mathcal{A}, X)$ for $\tau_{s}$. Then $\mu_{\alpha}(f) \rightarrow \mu(f)$ for each $f \in \mathcal{A}$, and since $\mu_{\alpha}(f) \in \overline{c o} f(S)$ for all $\alpha, \mu(f) \in \overline{c o} f(S)$. Therefore, $\mu \in M(\mathcal{A})$.

(2) Clearly, $\operatorname{co}(\epsilon(S)) \subset M(\mathcal{A})$. If there is a $\mu \in M(\mathcal{A})$ such that $\mu \notin \overline{c o}(\epsilon(S))$, the closure being taken in $\tau_{s}$, then there is an $f \in \mathcal{A}$ such that $\mu(f) \notin \overline{c o}(\epsilon(S) f)=$ $\overline{c o} f(S)$, which contradicts Definition 1.1.

(3) is obvious. 
The proof of the compactness of $M(\mathcal{A})$, if $\mathcal{A}$ satisfies the compactness condition, is similar to that of its counterpart in the following proposition, so we omit it.

Proposition 1.6. Let $\mathcal{A}$ be a linear subspace of $\mathcal{B}(S, X)$. Then the conclusions (1)-(3) of the previous proposition are true for $\tau_{w}$. Furthermore, if $\mathcal{A}$ is such that the range $f(S)$ of $f$ is weakly relatively compact in $X$ for each $f \in \mathcal{A}$, then $M(\mathcal{A})$ is $\tau_{w}$-compact.

Proof. Using Lemma 1.4, we can prove (1)-(3) in much the same way that (1)-(3) of Proposition 1.5 we proved.

We now show that $M(\mathcal{A})$ is $\tau_{w}$-compact when $\mathcal{A}$ satifies the weak compactness condition. For each $x^{*} \in X^{*}, M\left(x^{*} \mathcal{A}\right)$ is weak $^{*}$ compact $[1,2.1 .8]$. Therefore, the product space

$$
\prod:=\prod\left\{M\left(x^{*} \mathcal{A}\right): x^{*} \in X^{*}\right\}
$$

is compact in the product topology.

By Lemma 1.4, the mapping $\mu \rightarrow \varphi_{\mu}=\left\{\varphi_{\mu, x^{*}}: x^{*} \in X^{*}\right\}: M(\mathcal{A}) \rightarrow \prod$ is $1-1$, and it is homeomorphism when $M(\mathcal{A})$ has the topology $\tau_{w}$. To show that $M(\mathcal{A})$ is $\tau_{\boldsymbol{w}}$-compact, it suffices to show that the image of $M(\mathcal{A})$ in $\prod$ is closed.

Let $\varphi=\left\{\varphi_{x^{*}}: x^{*} \in X^{*}\right\} \in \prod$ and let the image $\left\{\varphi_{\mu_{\alpha}}\right\}$ of $\left\{\mu_{\alpha}\right\}$ converge to $\varphi$ in $\Pi$. We show that there is a $\mu \in M(\mathcal{A})$ such that $\varphi$ is the image of $\mu$ and $\mu_{\alpha} \rightarrow \mu$ in $\tau_{w}$.

Since $f(S)$ is weakly relatively compact in $X$ for each $f \in \mathcal{A}$, by the Krein-Smulian theorem [1, A.10] $\overline{c o} f(S)$ is weakly compact in $X$ for each $f \in \mathcal{A}$. Since $\mu_{\alpha}(f) \in \overline{c o} f(S)$ for all $\alpha$ and $x^{*} \mu_{\alpha}(f) \rightarrow \varphi_{x^{*}}\left(x^{*} f\right)$ for all $x^{*} \in X^{*}$, there is a $\mu(f) \in \overline{c o} f(S)$ such that $x^{*} \mu(f)=\varphi_{x^{*}}\left(x^{*} f\right)$ for all $x^{*} \in X^{*}$. The map $f \rightarrow \mu(f)$ is clearly linear, so $\mu \in M(\mathcal{A})$. Thus $\mu_{\alpha} \rightarrow \mu$ in $\tau_{w}$, and the proof is complete.

The following theorem shows that the definition of a mean in [2] is equivalent to that in $[3]$.

Theorem 1.7. A mapping $\mu: \mathcal{A} \rightarrow X$ is in $M(\mathcal{A})$ if and only if there is a unique $\varphi_{\mu} \in M\left(\overline{s p}\left(X^{*} \circ \mathcal{A}\right)\right)$ such that

$$
x^{*} \mu(f)=\varphi_{\mu}\left(x^{*} f\right) \quad\left(x^{*} \in X^{*}, f \in \mathcal{A}\right) .
$$

Proof. The sufficiency comes from the sufficiency in the first statement of Lemma 1.4.

Necessity. By Lemma 1.4, if $\mu$ is in $M(\mathcal{A})$, then for each $x^{*} \in X^{*}$ there is a $\varphi_{\mu, x^{*}}$ in $M\left(x^{*} \mathcal{A}\right)$ such that

$$
x^{*} \mu(f)=\varphi_{\mu, x^{*}}\left(x^{*} f\right) \quad(f \in \mathcal{A})
$$

We show first that $\varphi_{\mu, x^{*}}$ is independent of $x^{*} \in X^{*}$, i.e., if $x_{1}^{*}, x_{2}^{*} \in X^{*}$ and $f_{1}, f_{2} \in \mathcal{A}$ are such that $x_{1}^{*} f_{1}=x_{2}^{*} f_{2}$, then $\varphi_{\mu, x_{1}^{*}}\left(x_{1}^{*} f_{1}\right)=\varphi_{\mu, x_{2}^{*}}\left(x_{2}^{*} f_{2}\right)$.

Since $\mu \in M(\mathcal{A})$, by Proposition $1.6(2)$ there is a net $\left\{\sum_{s \in S} \lambda_{\alpha}(s) \epsilon(s)\right\}$ converging to $\mu$ for $\tau_{w}$; here each $\lambda_{\alpha}: S \rightarrow[0,1]$ has finite support and satisfies $\sum_{s \in S} \lambda_{\alpha}(s)=1$. Next, $x_{1}^{*}\left(\sum_{s \in S} \lambda_{\alpha}(s) f_{1}(s)\right)=x_{2}^{*}\left(\sum_{s \in S} \lambda_{\alpha}(s) f_{2}(s)\right)$ because $x_{1}^{*} f_{1}=x_{2}^{*} f_{2}$, so 


$$
\begin{aligned}
\varphi_{\mu, x_{i}^{*}}\left(x_{1}^{*} f_{1}\right)=x_{1}^{*} \mu\left(f_{1}\right) & =\lim _{\alpha} x_{1}^{*} \sum_{s \in S} \lambda_{\alpha}(s) f_{1}(s) \\
& =\lim _{\alpha} x_{2}^{*} \sum_{s \in S} \lambda_{\alpha}(s) f_{2}(s)=x_{2}^{*} \mu\left(f_{2}\right)=\varphi_{\mu, x_{2}^{*}}\left(x_{2}^{*} f_{2}\right) .
\end{aligned}
$$

Therefore we can define $\varphi_{\mu}$ for $\sum_{\imath=1}^{m} \alpha_{\imath} x_{\imath}^{*} f_{\imath} \in s p\left(X^{*} \circ \mathcal{A}\right)$ by

$$
\varphi_{\mu}\left(\sum_{i=1}^{m} \alpha_{\imath} x_{\imath}^{*} f_{\imath}\right)=\sum_{i=1}^{m} \alpha_{\imath} \varphi_{\mu, x_{i}^{*}}\left(x_{\imath}^{*} f_{\imath}\right) .
$$

It is easy to see that $\varphi_{\mu}$ is in $M\left(s p\left(X^{*} \circ \mathcal{A}\right)\right)$. Therefore $\varphi_{\mu}$ has a unique extension to $\overline{s p}\left(X^{*} \circ \mathcal{A}\right)$ and satisfies $(1.1)$.

The uniqueness is clear. The proof is finished.

By Theorem 1.7, we can write $\varphi_{\mu}$ for $\varphi_{\mu, x^{*}}$ in Lemma 1.4 .

\section{§. Introversion and Semigroups of Vector-Valued Means}

Definition 2.1. Let $\mathcal{A}$ be a translation invariant linear subspace of $\mathcal{B}(S, X)$. For a linear map $\mu$ from $\mathcal{A}$ to $X$, define the left introversion operator $T_{\mu}: \mathcal{A} \rightarrow \mathcal{B}(S, X)$ by

$$
T_{\mu} f(s)=\mu\left(L_{s} f\right) \quad(f \in \mathcal{A}, s \in S)
$$

and analogously define the right introversion operator $U_{\mu}: A \rightarrow \mathcal{B}(S, X)$ by

$$
U_{\mu} f(s)=\mu\left(R_{s} f\right) \quad(f \in \mathcal{A}, s \in S) .
$$

If $T_{\mu} \mathcal{A} \subset \mathcal{A}$ for all $\mu \in M(\mathcal{A})$, we will say that $\mathcal{A}$ is left introverted; we will say that $A$ is right introverted if $U_{\mu} \mathcal{A} \subset \mathcal{A}$. $\mathcal{A}$ is introverted if it is both left and right introverted.

A semitopological semigroup $S$ is a semigroup and a Hausdorff topological space in such a way that multiplication is separately continuous, i.e., the maps $s \rightarrow t s$ and $s \rightarrow s t$ from $S$ into $S$ are continuous for all $t \in S . \mathcal{C}(S, X)$ denotes the Banach space of all continuous members of $\mathcal{B}(S, X)$.

Example 2.2. $\mathcal{C}(S, X)$ is introverted if $S$ is a compact semitopological semigroup.

For $\mu \in M(\mathcal{C}(S, X))$ and $f \in \mathcal{C}(S, X)$, we must show that $T_{\mu} f$ and $U_{\mu} f$ are continuous.

Let $g \in \mathcal{C}(S)$ and let $x \in X . g(\cdot) x \in \mathcal{C}(S, X)$. Theorem 1.7 implies that $\mu(g(\cdot) x)=$ $\varphi_{\mu}(g) x$ and $T_{\mu}(g(\cdot) x)=T_{\varphi_{\mu}}(g) x$. Therefore $T_{\mu}(g(\cdot) x) \in \mathcal{C}(S, X)$ since $T_{\varphi_{\mu}}(g) \in \mathcal{C}(S)[1$, 2.2.5]. Note the fact that $\mathcal{C}(S, X)=\overline{s p}\{g(\cdot) x: g \in \mathcal{C}(S), x \in X\}$ since $S$ is compact. For $\epsilon>0$ there is $p(\cdot)=\sum_{\imath=1}^{n} f_{\imath}(\cdot) x_{\imath}$, where $f_{\imath} \in \mathcal{C}(S)$ and $x_{\imath} \in X, i=1,2, \cdots, n$, such that

$$
\|f-p\|<\epsilon
$$

Now $p \in \mathcal{C}(S, X)$ and

$$
\left\|T_{\mu} f-T_{\mu} p\right\|=\max _{s \in S}\left\|\mu\left(L_{s}(f-p)\right)\right\| \leq\|f-p\|<\epsilon .
$$

Therefore $T_{\mu} f \in \mathcal{C}(S, X)$.

Similarly $U_{\mu} f \in \mathcal{C}(S, X)$. The proof is finished. 
Proposition 2.3. Let $\mathcal{A}$ bc a translation invariant linear subspace of $\mathcal{B}(S . X)$ containing the constant functions and let $\epsilon: S \rightarrow M(\mathcal{A})$ be the evaluation mapping. Then

(1) for each $\mu \in M(\mathcal{A}), T_{\mu}: \mathcal{A} \rightarrow \mathcal{B}(S, X)$ is a bounded linear transformation with $\left\|T_{\mu}\right\| \leq\|\mu\|$

(2) the mapping $\mu \rightarrow T_{\mu}: M(\mathcal{A}) \rightarrow \mathcal{L}(\mathcal{A}, \mathcal{B}(S, X))$ is a bounded transformation;

(3) if $\mu \in M(\mathcal{A})$, then $T_{\mu}(x)=x, x \in X$;

(4) for all $s \in S$ and $\mu \in M(\mathcal{A})$

$$
\begin{aligned}
T_{\mu} L_{s} & =L_{s} T_{\mu} \\
T_{\mu} R_{s} & =T_{R_{s} \mu} \\
T_{\varepsilon(s)} & =R_{s},
\end{aligned}
$$

where $R_{s}^{*}: M(\mathcal{A}) \rightarrow M(\mathcal{A})$ is the adjoint of $R_{s}$;

(5) if $f \in \mathcal{A}$, then $\left\{T_{\mu} f: \mu \in M(\mathcal{A})\right\}$ is the closure in $\mathcal{B}(S, X)$ of $\operatorname{co}\left(R_{S} f\right)$ in the topology of pointwise convergence on $S$.

The proof of the proposition above is like that for $[1,2.2 .3]$, so we omit it.

Definition 2.4. Let $\mathcal{A}$ be a translation invariant linear subspace of $\mathcal{B}(S, X)$ containing the constant functions, and define

$$
Z_{T}=\left\{\nu \in \mathcal{L}(\mathcal{A}, X): T_{\nu} \mathcal{A} \subset \mathcal{A}\right\}
$$

and

$$
Z_{U}=\left\{\mu \in \mathcal{L}(\mathcal{A}, X): U_{\mu} \mathcal{A} \subset \mathcal{A}\right\}
$$

If $\mu \in \mathcal{L}(\mathcal{A}, X)$ and $\nu \in Z_{T}$, define $\mu \nu: \mathcal{A} \rightarrow X$ by

$$
\mu \nu(f)=\mu\left(T_{\nu} f\right) \quad(f \in \mathcal{A}) .
$$

If $\mu \in Z_{U}$ and $\nu \in \mathcal{L}(\mathcal{A}, X)$, define $\mu * \nu: \mathcal{A} \rightarrow X$ by

$$
\mu * \nu(f)=\nu\left(U_{\mu} f\right) \quad(f \in \mathcal{A}) .
$$

Definition 2.5. An admissible subspace $\mathcal{A}$ of $\mathcal{B}(S, X)$ is a norm closed, translation invariant, left introverted subspace of $\mathcal{B}(S, X)$ containing the constant functions. In the case that $X=\mathbb{C}$, an admissible subspace $\mathcal{A} \subset \mathcal{B}(S)$ is also required to be conjugate closed.

Let $S$ be a semigroup. Define $\rho_{t}: S \rightarrow S$ and $\lambda_{t}: S \rightarrow S$ by

$$
\rho_{t}=s t, \quad \lambda_{t}=t s \quad(s \in S) .
$$

$S$ is called a right topological semigroup if it is a topological space and $\rho_{t}$ is continuous for all $t \in S$. Set

$$
\Lambda(S)=\left\{s \in S: \lambda_{s} \text { is continuous }\right\} .
$$

An affine semigroup $S$ is a semigroup and a convex subset of a vector space in such a way that $\rho_{t}$ and $\lambda_{t}$ are affine mappings for each $t \in S$. The requirement that $\rho_{t}$ and $\lambda_{t}$ be affine means that if $r, s \in S$ and $a, b \in[0,1]$ with $a+b=1$ then

$$
(a r+b s) t=a r t+b s t \text { and } t(a r+b s)=a t r+b t s,
$$


where $(+)$ denotes vector addition.

The following lemma summarizes the properties of the operation $(\mu, \nu) \rightarrow \mu \nu$. The proof is similar to that of $[1,2.2 .9]$. We omit the statements of the corresponding properties of the operation $(\mu, \nu) \rightarrow \mu * \nu$.

Lemma 2.6. Let $\mathcal{A}$ be as in Definition 2.4 and let $\epsilon: \mathcal{A} \rightarrow X$ be the evaluation mapping. Then

(1) $Z_{T}$ is a linear subspace of $\mathcal{L}(\mathcal{A}, X)$ containing $\epsilon(S)$;

(2) $\mu \nu \in \mathcal{L}(\mathcal{A}, X)$ for all $\mu \in \mathcal{L}(\mathcal{A}, X)$ and $\nu \in Z_{T}$;

(3) if $\mu \in \mathcal{L}(\mathcal{A}, X), \nu \in Z_{T}$ and $s \in S$, we have

$$
\begin{aligned}
T_{\mu \nu} & =T_{\mu} \circ T_{\nu}, \\
\varepsilon(s) \nu & =L_{s}^{*} \nu, \\
\mu \varepsilon(s) & =R_{s}^{*} \mu, \text { and } \\
\|\mu \nu\| & \leq\|\mu\|\|\nu\|,
\end{aligned}
$$

where $L_{s}^{*}: M(\mathcal{A}) \rightarrow M(\mathcal{A})$ is the adjoint of $L_{s} ;$

(4) $Z_{T}$ is a right topological semigroup.

The following result is essentially a consequence of the preceding lemma and Propositions 1.5 and 1.6.

\section{Theorem 2.7.}

(1) If $\mathcal{A}$ is an admissible subspace of $\mathcal{B}(S, X)$, then for $\tau_{s}$ or $\tau_{w}$, and multiplication $(\mu, \nu) \rightarrow \mu \nu, M(\mathcal{A})$ is a right topological affine subsemigroup of $\mathcal{L}(\mathcal{A}, X)$, $\operatorname{co}(\epsilon(S)) \subset \Lambda(M(\mathcal{A}))$ and $\epsilon: S \rightarrow M(\mathcal{A})$ is a homomorphism.

(2) If we also assume that $f(S)$ is (weakly) relatively compact for all $f \in \mathcal{A}$, then $M(\mathcal{A})$ is also compact for $\left(\tau_{w}\right) \tau_{s}$.

Let $S$ be a compact semitopological semigroup. By Example 2.2, $\mathcal{C}(S, X)$ is introverted. Hence $\mu \nu, \mu * \nu \in M(\mathcal{C}(S, X))$; indeed, they are equal.

Proposition 2.8. Let $S$ be a compact semitopological semigroup and let $\mathcal{A}=\mathcal{C}(S, X)$.

Then

(1) $\mu \nu=\mu * \nu$ for all $\mu, \nu \in M(\mathcal{A})$;

(2) for $\tau_{s}$ and multiplication $(\mu, \nu) \rightarrow \mu \nu, M(\mathcal{A})$ is a compact semitopological affine semigroup;

(3) if $S$ is also a topological semigroup, so is $M(\mathcal{A})$ in $\tau_{s}$.

Proof. (1). Note that $\varphi_{\mu} \varphi_{\nu}=\varphi_{\mu} * \varphi_{\nu}[1,2.2 .12$ (a)]. Similar to the proof of Example 2.2, we have, for $g \in \mathcal{C}(S)$ and $x \in X$,

$$
\mu \nu(g(\cdot) x)=\mu\left(T_{\nu} g(\cdot) x\right)=\varphi_{\mu}\left(T_{\varphi_{\nu}} g\right) x=\varphi_{\mu} \varphi_{\nu}(g) x=\varphi_{\mu} * \varphi_{\nu}(g) x=\mu * \nu(g(\cdot) x) .
$$


Therefore

$$
\mu \nu(f)=\mu * \nu(f) \quad(f \in \mathcal{C}(S, X))
$$

i.e., $\mu \nu=\mu * \nu$.

(2) is a consequence of (1) and Theorem 2.7 (1).

To verify (3), we need to show that if $\mu_{\alpha} \rightarrow \mu$ and $\nu_{\alpha} \rightarrow \nu$ for $\tau_{s}$ then $\mu_{\alpha} \nu_{\alpha} \rightarrow \mu \nu$ for $\tau_{s}$. Note that $\varphi_{\mu_{\alpha}} \varphi_{\nu_{\alpha}}(g) \rightarrow \varphi_{\mu} \varphi_{\nu}(g)$ for every $g \in \mathcal{C}(S)[1,2.2 .12$ (c)]. Now, for $x \in X$,

$$
\mu_{\alpha} \nu_{\alpha}(g(\cdot) x)=\varphi_{\mu_{\alpha}} \varphi_{\nu_{\alpha}}(g) x \rightarrow \varphi_{\mu} \varphi_{\nu}(g) x=\mu \nu(g(\cdot) x)
$$

Again using the fact that $\mathcal{C}(S, X)=\overline{s p}\{g(\cdot) x: g \in \mathcal{C}(S), x \in X\}$, we have $\mu_{\alpha} \nu_{\alpha}(f) \rightarrow \mu \nu(f)$ for every $f \in \mathcal{C}(S, X)$.

\section{$\S$ 3. Invariant Vector-Valued Means}

$S$ denotes a semigroup which need not have an identity and $\mathcal{A}$ denotes a linear subspace of $\mathcal{B}(S, X)$ containing the constant functions. Let $\operatorname{LIM}(\mathcal{A})(R I M(\mathcal{A}))$ denotes the set of left (right) invariant means on $\mathcal{A}$. $\mathcal{A}$ is said to be left (right) amenable if $\operatorname{LIM}(\mathcal{A}) \neq \phi$ $(R I M(\mathcal{A}) \neq \phi)$. If $\mathcal{A}$ is translation invariant, we set

$$
\operatorname{IM}(\mathcal{A})=\operatorname{LIM}(\mathcal{A}) \cap \operatorname{RIM}(\mathcal{A})
$$

and call members of $\operatorname{IM}(\mathcal{A})$ invariant means. $\mathcal{A}$ is said to be amenable if $I M(\mathcal{A}) \neq \phi$.

As in the scalar case, we have the following proposition, whose proof is similar to that of $[1,2.3 .5]$; so we omit it.

Proposition 3.1. Let $\mathcal{A}$ be an admissible subspace of $\mathcal{B}(S, X)$ and let $\epsilon: S \rightarrow \mathcal{L}(\mathcal{A}, X)$ be the evaluation mapping.

(1) $\operatorname{LIM}(\mathcal{A})$ is the set of right zeros of $M(\mathcal{A})$; hence if $\mathcal{A}$ is left amenable, then $\operatorname{LIM}(\mathcal{A})$ is a closed ideal of $M(\mathcal{A})$ contained in every right ideal.

(2) If $\mathcal{A}$ is right amenable, then $\operatorname{RIM}(\mathcal{A})$ is a closed left ideal of $M(\mathcal{A})$.

Corollary 3.2. Let $\mathcal{A}$ be an admissible subspace of $\mathcal{B}(S, X)$. If $\mathcal{A}$ is left and right amenable, then it is amenable.

Proof. If $\mu \in \operatorname{LIM}(\mathcal{A})$ and $\nu \in R I M(\mathcal{A})$, then $\mu \nu \in I M(\mathcal{A})$.

Corollary 3.3. Let $\mathcal{A}$ be an admissible right introverted subspace of $\mathcal{B}(S, X)$ such that $\mu \nu=\mu * \nu$ for all $\mu, \nu \in M(\mathcal{A})$. Then $\mathcal{A}$ has at most one invariant mean.

Proof. By the proposition and its right introverted analog, if $\mu, \nu \in I M(\mathcal{A})$, then $\nu=$ $\mu \nu=\mu * \nu=\mu$.

Theorem 3.4. Let $\mathcal{A}$ be an admissible subspace of $\mathcal{B}(S, X)$ such that, for each $f \in \mathcal{A}$, the range $f(S)$ of $f$ is relatively weakly compact. Let $K(f)$ denote the closure in $\mathcal{B}(S, X)$ of $c o\left(R_{S} f\right)$ for the pointwise topology. The following assertions are equivalent: 
(1) $\mathcal{A}$ is left amenable:

(2) for cach $f \in \mathcal{A} . K(f)$ contains a constant function;

(3) for cach $f \in \mathcal{A}$. and $s \in S, 0 \in K\left(f-L_{s} f\right)$.

Furthermore. if (1) holds then. for cach $f \in \mathcal{A} .\{\mu(f): \mu \in L I M(\mathcal{A})\}$ is the set of constant functions in $K(f)$.

Proof. We omit the proofs that $(1) \Rightarrow(2) \Rightarrow(3)$ which do not use weak compactenss hypothesis. Here we show that $(3) \Rightarrow(1)$.

For cach $f \in \mathcal{A}$ and $s \in S$, let

$$
M(f, s)=\left\{\mu \in M(A): T_{\mu}\left(f-L_{s} f\right)=0\right\} .
$$

The sets $M(f, s)$ are $\tau_{w}$-closed, and therefore $\tau_{w}$-compact. For, let $\left\{\mu_{\alpha}\right\} \subset M(f, s)$ converge to $\mu \in M(\mathcal{A})$. We want to show that $\mu \in M(f, s)$, i.e.,

Note that

$$
T_{\mu}\left(f-L_{s} f\right)=0 \text {. }
$$

$$
T_{\mu}\left(f-L_{s} f\right)(t)=\mu\left(L_{t} f-L_{t s} f\right) \quad(t \in S)
$$

and $\mu_{\alpha}\left(L_{t} f-L_{t s} f\right)=T_{\mu_{\alpha}}\left(f-L_{s} f\right)(t)=0$ for all $\alpha$. Since $\mu_{\alpha}\left(L_{t} f-L_{t s} f\right) \rightarrow \mu\left(L_{t} f-L_{t s} f\right)$ weakly, $\mu\left(L_{t} f-L_{t s} f\right)=0$. That is, $T_{\mu}\left(f-L_{s} f\right)=0$.

As in the proof of $[1,2.3 .11]$, we can show that the family $\{M(f, s): f \in \mathcal{A}, s \in S\}$ has the finite intersection property. By Proposition $1.6 M(\mathcal{A})$ is $\tau_{w}$-compact. So

$$
\bigcap\{M(f, s): f \in \mathcal{A}, s \in S\} \neq \phi .
$$

Let $\mu$ be any member of this intersection, then $\mu^{2} \in L I M(\mathcal{A})$.

Let $S$ be a group and let $\mathcal{A}$ be a linear subspace of $\mathcal{B}(S, X)$. For each $f \in A$ define $\tilde{f}$ : $S \rightarrow X$ by

$$
\tilde{f}(s)=f\left(s^{-1}\right) \quad(s \in S),
$$

and set

$$
\tilde{\mathcal{A}}=\{\tilde{f}: f \in \mathcal{A}\}
$$

If $\mu \in M(\mathcal{A})$, define $\tilde{\mu} \in M(\tilde{\mathcal{A}})$ by

$$
\tilde{\mu}(\tilde{f})=\mu(f) \quad(f \in \mathcal{A}) .
$$

If $\tilde{\mathcal{A}}=\mathcal{A}$ and $\tilde{\mu}=\mu$, then $\mu$ is said to be inversion invariant.

Theorem 3.5. Let $G$ be a compact Hausdorff topological group. Then $\mathcal{C}(G, X)$ has a unique invariant mean $\mu$. Furthermore $\mu$ is inversion invariant.

Proof. The mean $\mu$ can be expressed as

$$
\mu(f)=\int_{G} f d \nu \quad(f \in \mathcal{C}(G, X)),
$$

where $\nu$ is normalized Haar measure on $G$; the properties of $\mu$ follows from those of $\nu$. 
The scalar version of the next theorem is $[1,2.3 .14]$; a similar result has appeared in [3], but there $S$ is required to have an identity. A small modification of the proof of $[1$, 2.3.14] yields a proof of the present theorem.

Theorem 3.6. Let $S$ be a compact Hausdorff semitopological semigroup. Then the following asscrtions hold:

(1) $\mathcal{C}(S, X)$ is left (respectively right) amenable if and only if $S$ has a unique minimal right (respectively, left) ideal;

(2) $\mathcal{C}(S, X)$ is amenable if and only if the minimal ideal of $S$ is a compact topological group.

\section{§4. Vector-Valued Weakly Almost Periodic Functions}

Let $S$ be a semitopological semigroup; we do not assume $S$ has an identity. Let $\mathcal{W A P}(S, X)$ consist of those members $f$ of $\mathcal{C}(S, X)$ for which the right orbit $R_{S} f=\left\{R_{s} f:\right.$ $s \in S\}$ is weakly relatively compact in $\mathcal{C}(S, X)$.

With a proof similar to that for $[1,4.2 .5]$, one sees that the space $\mathcal{W A P}(S, X)$ is a closed translation invariant subspace of $\mathcal{C}(S, X)$. When $X=\mathbb{C}, \mathcal{W} \mathcal{A}(S, X)$ is just $\mathcal{W A P}(S)$, the $C^{*}$-algebra of weakly almost periodic functions on $S$. We note that

$$
x^{*} \circ \mathcal{W} \mathcal{A} \mathcal{P}(S, X)=\mathcal{W} \mathcal{A} \mathcal{P}(S) \quad\left(x^{*} \in X^{*}, x^{*} \neq 0\right)
$$

Recall that $\epsilon: S \rightarrow \mathcal{L}(\mathcal{A}, X)$ is the evaluation mapping $\epsilon(s) f=f(s), f \in \mathcal{W} \mathcal{A P}(S, X)$. When $X=\mathbb{C}$ we denote this mapping by $\epsilon^{\prime}$.

Let $a S^{\mathcal{W A P}}$ denote the $w^{*}$ closure in $\mathcal{W} \mathcal{A P}(S)^{*}$ of $c o \epsilon^{\prime}(S) ; a S^{\mathcal{W A P}}$ is a compact affine semitopological semigroup $[1,4.2 .11]$.

Theorem 4.1. Let $S$ be a semitopological semigroup and let $\mathcal{A}=\mathcal{W} \mathcal{A} \mathcal{P}(S, X)$. The following assertions hold:

(1) $\mathcal{A}$ is an admissible subspace of $\mathcal{B}(S, X)$;

(2) for $\tau_{w}$ and multiplication $(\mu, \nu) \rightarrow \mu \nu, M(\mathcal{A})$ is an affine semitopological semigroup;

(3) if $f(S)$ is weakly relatively compact in $X$ for each $f \in \mathcal{A}$, then $M(\mathcal{A})$ is $\tau_{w}$-compact; in this case $\mathcal{A}$ is left amenable if and only if $\mathcal{W A P}(S)$ is left amenable.

Proof. (1) Since $\mathcal{A}$ is a closed translation invariant subspace of $\mathcal{C}(S, X)$, to show that $\mathcal{A}$ is admissible we need to show that $\mathcal{A}$ is left introverted, i.e., if $f \in \mathcal{A}$ then $T_{\mu} f \in \mathcal{A}$ for all $\mu \in M(\mathcal{A})$.

Define $V: M(\mathcal{A}) \rightarrow \mathcal{B}(S, X)$ by

$$
V(\mu)=T_{\mu} f \quad(\mu \in M(\mathcal{A}))
$$

By Proposition 2.3 (5)

$$
V(M(\mathcal{A}))=\overline{c o}\left(R_{S} f\right)
$$


the closure being taken in the pointwise topology. Since $f \in \mathcal{A}, \operatorname{co}\left(R_{S} f\right)$ is weakly relatively compact in $\mathcal{A}$; in view of $(4.1)$ this implies that $V(M(\mathcal{A}))$ is the weak closure in $\mathcal{A}$ of $\operatorname{co}\left(R_{S} f\right)$. So $T_{\mu} f \in \mathcal{A}$ for all $\mu \in M(\mathcal{A})$.

(2) By Theorem $2.7(1)$, for $\tau_{w}$ and multiplication $(\mu, \nu) \rightarrow \mu \nu, M(\mathcal{A})$ is a right topological affine semigroup. It follows from Theorem 1.7 that the mapping $\Pi: \mu \rightarrow \varphi_{\mu}$ is a $\tau_{w}-w^{*}$ homeomorphism of $M(\mathcal{A})$ into $a S^{\mathcal{W A P}}$. Since $x^{*} \nu(f)=\varphi_{\nu}\left(x^{*} f\right)$ for $f \in \mathcal{A}$ and $x^{*} \in X^{*}, x^{*}\left(T_{\nu} f\right)=T_{\varphi_{\nu}}\left(x^{*} f\right)$. It follows that $\varphi_{\mu \nu}=\varphi_{\mu} \varphi_{\nu}$. Since $\Pi(\mu \nu)=\varphi_{\mu \nu}, \Pi$ is a homomorphism too. So $M(\mathcal{A})$ is an affine semitopological semigroup because $a S^{\mathcal{W A P}}$ is.

(3) When $\mathcal{A}$ satisfies the compactness condition, the $\tau_{w}$-compactness of $M(\mathcal{A})$ is a consequence of Theorem $2.7(2)$. In this case, $M(\mathcal{A}) \cong a S^{\mathcal{W A P}}$. So we get the last statement.

The proof is complete.

Remark 4.2. For $f \in \mathcal{W} \mathcal{A} \mathcal{P}(S, X)$, in general $f(S) \subset X$ is not weakly relatively compact. However, if $S$ admits an identity, it follows from the double limit property (e.g., [2, Theorem 3]) that $f(S)$ is weakly relatively compact. Of course, if $X$ is reflexive then $f(S)$ is weakly relatively compact.

Theorem 4.3. For a compact semitopological semigrooup $S, \mathcal{W A} \mathcal{P} S, X=\mathcal{C}(S, X)$.

The theorem holds because the facts of $\mathcal{C}(S, X)=\overline{s p}\{f(\cdot) x: f \in \mathcal{C}(s), x \in X\}$ and $\mathcal{W} \mathcal{A} \mathcal{P}(S)=\mathcal{C}(S)[1,4.2 .9]$.

ACKNOWLEDGEMENT. The contents of this paper is a part of the author's Ph.D. work under the supervision of Professor P. Milnes at the University of Western Ontario. The author would like to acknowledge his indebtedness to his supervisor, Professor P. Milnes, for advice and encouragement given while this paper was being written. The author also wishes to thank to the referee for suggesting simplified proofs of Example 2.2 and Proposition 2.8. This led to Theorem 4.3.

\section{REFERENCES}

1. J.F. Berglund, H.D. Junghenn and P. Milnes, Analysis on Semigroups: Functıon Spaces, Compactifications, Representations, Wiley, New York, 1989.

2. P. Milnes, On vector-valued weakly almost periodic functions, J. London Math. Soc.(2), 22 (1980), 467-472.

3. S. Goldberg and P. Irwin, Weakly almost periodic vector valued functions, Dissertationes Math., 157(1979). 


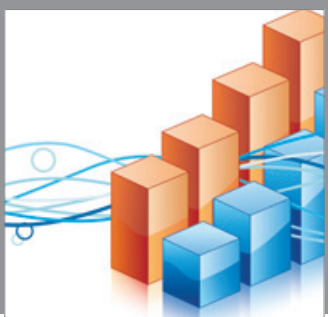

Advances in

Operations Research

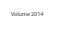

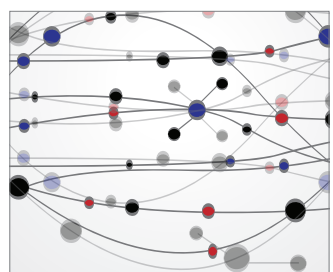

\section{The Scientific} World Journal
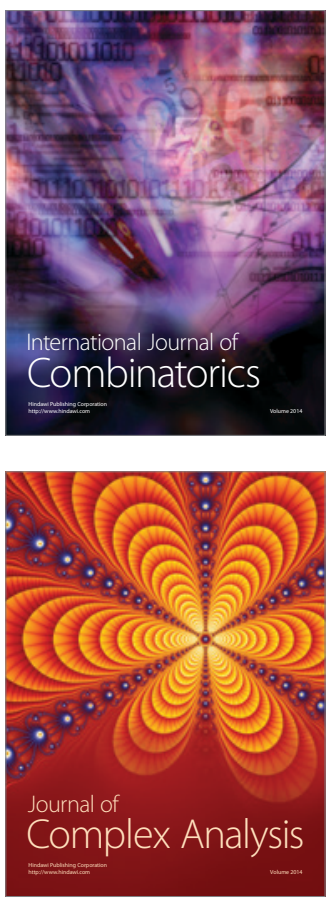

International Journal of

Mathematics and

Mathematical

Sciences
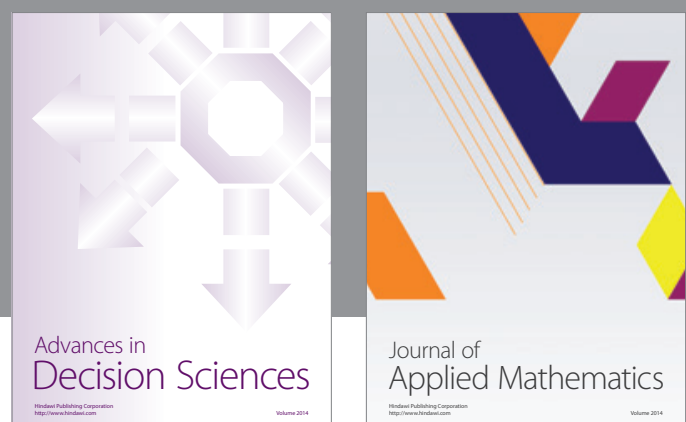

Journal of

Applied Mathematics
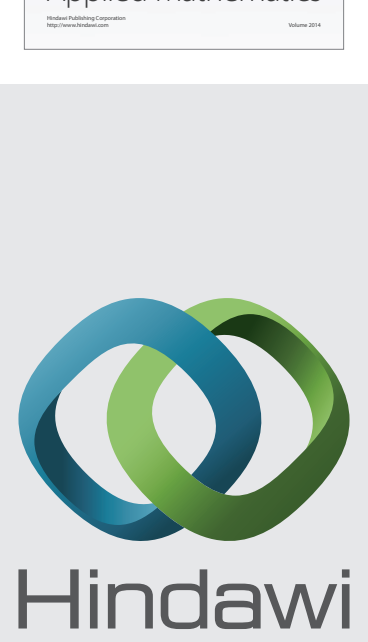

Submit your manuscripts at http://www.hindawi.com
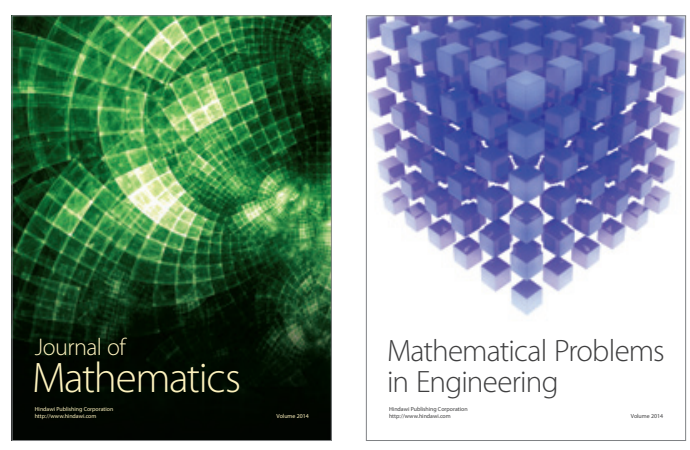

Mathematical Problems in Engineering
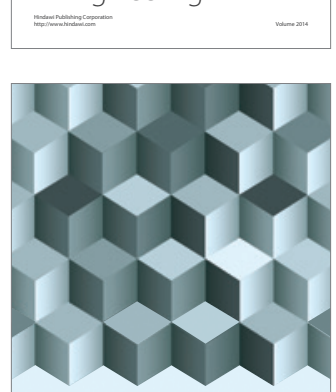

Journal of

Function Spaces
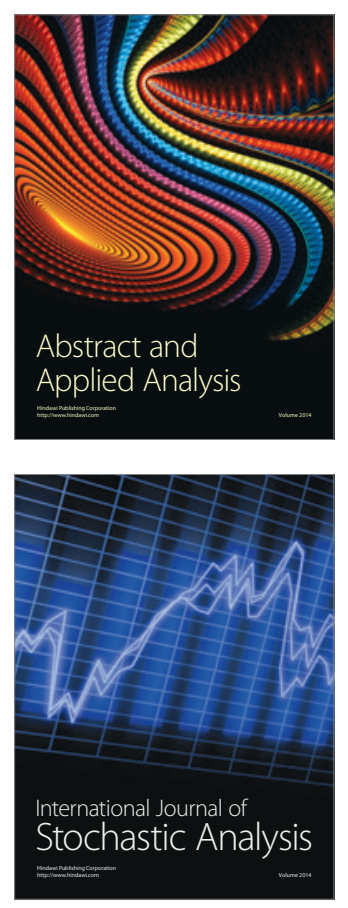

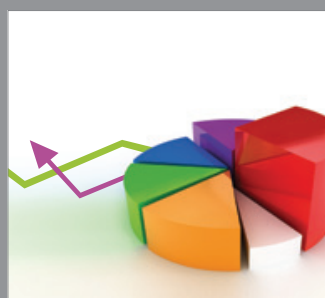

ournal of

Probability and Statistics

Promensencen
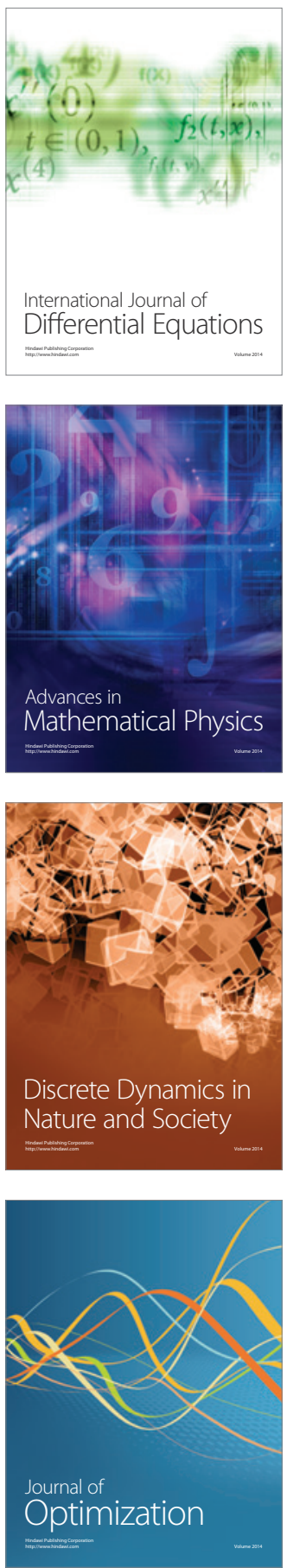